function-associated antigen 1), the principal lymphocyte integrin that mediates adhesion to intercellular adhesion molecule 1 expressed on the surface of endothelial cells ${ }^{1}$. The $\mathrm{C}$ terminus, $\mathrm{N}$ terminus and Ras (or Rap1) binding domain of RAPL are all required for this association. These data led to the model that RAPL is recruited by Rap 1 to associate with and activate $\alpha_{L} \beta_{2}$ (Fig. 1). This model is now strongly reinforced by the phenotype of RAPL knockout mice: isolated $\mathrm{B}$ and $\mathrm{T}$ lymphocytes from RAPL-deficient mice are much less adherent to intercellular adhesion molecule 1 and do not show the characteristic cell polarization and patchy appearance of integrins after stimulation with cytokines. In addition, T lymphocytes show impaired transmigration through an endothelial layer in the presence of shear stress, whereas both RAPL and constitutively active Rap1 induce transmigration. In the RAPL knockout mice, B and T lymphocytes as well as dendritic cells show a substantial impairment in their homing to the target organs, such as lymph nodes and spleen, most likely as a consequence of the defect in integrin-mediated cell adhesion. These results establish the importance of RAPL in the regulation of integrin adhesiveness and strongly support the idea that RAPL is a chief effector of Rap1. These data contribute a substantial breakthrough in both the understanding of inside-out signaling to integrins as well as for understanding the molecular mechanism of how Rap1 regulates integrin function.

How does RAPL regulate integrin function? Because RAPL was found in a complex with $\alpha_{L} \beta_{2}$ (ref. 1), RAPL might bind directly to integrins, thereby promoting subsequent ligand binding and clustering. T lymphocytes and splenic dendritic cells from RAPL knockout mice also show reduced adhesion to fibronectin via the integrin $\alpha_{4} \beta_{1}$ (VLA-4) ${ }^{2}$. Although direct binding was not demonstrated between RAPL and $\alpha_{L} \beta_{2}$, RAPL may well bind to the cytoplasmic tails of various integrin $\alpha$-subunits. RAPL overexpression also induces the clustering of the cytokine receptor CXCR4 at the leading edge ${ }^{1}$. While the effects on CXCR 4 and $\alpha_{4} \beta_{1}$ may be a consequence of $\alpha_{L} \beta_{2}$ activation, RAPL may actually control a more fundamental process that influences the mobility of a variety of cell surface receptors, for example by affecting membrane or cytoskeletal rigidity or linkage of receptors to the cytoskeleton. This hypothesis would be compatible with the observation that Rap 1 also regulates the adhesion properties of other classes of adhesion molecules, including cadherins ${ }^{9,10}$.

For Rap1, the story is not over yet. Most small GTPases have a plethora of effectors to mediate their functions, and Rap1 will be no exception. RAPL is expressed principally in lymphoid organs and it can therefore be expected that other effectors mediate Rap1 effects in nonlymphoid cells, perhaps the ubiquitously expressed splice variant of Rassf5 Norela. Many other proteins also interact with Rap1. For example, in drosophila, the interaction between Rap1 and the ortholog of mammalian AF6, canoe, is responsible for cell migration during dorsal closure ${ }^{11}$.
Rap1 interacts with and activates ARAP3, a GTPase-activating protein for RhoA, and Arf6 (ref. 12), two distant 'cousins' of Rap that regulate actin remodeling and vesicular trafficking. Involvement of Rho family GTPases in the effects of Rap is not immediately apparent. However, Rap is localized mainly on intracellular vesicles and must be transported to the cell surface $^{7}$, a task that may involve Arf6 function. Indeed, the spatial and temporal control of Rap and its signaling partners by vesicular trafficking may be critical in engagement of the adhesion signaling machinery rapidly and at appropriate sites in the cell. Although the details of RAPL's involvement have yet to be solved, RAPL has been 'fingered as a prime suspect' in Rap-dependent immune responses.

1. Katagiri, K., Maeda, A., Shimonaka, M. \& Kinashi, T. Nat. Immunol. 4, 741-748 (2003).

2. Katagiri, K. et al. Nat. Immunol. 5, 1045-1051 (2004)

3. Hogg, N. et al. Immunol. Rev. 186, 164-171 (2002).

4. Bos, J.L. et al. Biochem. Soc. Trans. 31, 83-86 (2003).

5. Sebzda, E., Bracke, M., Tugal, T., Hogg, N. \& Cantrell, D.A. Nat. Immunol. 3, 251-258 (2002).

6. Kinbara, K., Goldfinger, L.E., Hansen, M., Chou, F.L. \& Ginsberg, M.H. Nat. Rev. Mol. Cell Biol. 4, 767-776 (2003).

7. Dustin, M.L., Bivona, T.G. \& Philips, M.R. Nat. Immunol. 5, 363-372 (2004).

8. Vavvas, D., Li, X., Avruch, J. \& Zhang, X.F. J. Biol. Chem. 273, 5439-5442 (1998).

9. Knox, A.L. \& Brown, N.H. Science 295, 1285-1288 (2002).

10. Price, L.S. et al. J. Biol. Chem. 279, 35127-35132 (2004).

11. Boettner, B. et al.. Genetics 165, 159-169 (2003).

12. Krugmann, S. et al. Mol. Cell 9, 95-108 (2002).

\title{
Unmasking connections in transmembrane immune signaling
}

\author{
Jamey D Marth
}

\section{The B cell transmembrane glycoprotein modulates immune signaling. Mice containing specific $C d 22$ mutations show that the extracellular glycan-binding function is regulatory and indicate unexpected complexity in CD22-ligand interactions.}

Cell surface 'real estate' is frequently remodeled. Neighbors come and go, and their varied interactions emit regulatory signals across the membrane and into

Jamey D. Marth is with the Howard Hughes Medical Institute and Department of Cellular and Molecular Medicine at the University of California San Diego, La Jolla, California, 92093, USA. e-mail: jmarth@ucsd.edu the cell. Amid today's more chic intracellular listings, it is a buyer's market in the study of extracellular features that contribute to signal transduction. Nevertheless, equity has been increasing among a class of mammalian transmembrane molecules of the immunoglobulin superfamily called Siglecs. The defining characteristic of the Siglec family is an extracellular domain that binds to glycan structures bearing specific sialic acid linkage termini produced during protein glycosylation in the Golgi apparatus ${ }^{1}$. In this issue of Nature Immunology, Poe et al. report that the Siglec protein CD22 has both Siglec-dependent and Siglec-independent activities that modulate B cell physiology and immune function ${ }^{2}$.

CD22 is a prototype of the Siglec family, but it has been known longer as a B cellspecific transmembrane glycoprotein containing an intracellular phosphorylation motif called an ITIM that participates in 
B lymphocyte activation ${ }^{3,4}$ (Fig. 1). These structural attributes have engaged the interest of many researchers considering the potential for Siglec-dependent activities among CD22dependent processes. After all, noticeably embedded in CD22 structure is the intriguing possibility that two types of topologically separated post-translational protein modifications, glycosylation and phosphorylation, collaborate to determine immunological signals that cross the membrane and regulate immunity. Such 'cross-culture' collaboration seems even more likely now.

In an age when increased value in a protein is often born from the outcomes of its depletion in mouse gene-targeting experimentation, CD22 underwent a complicated birth, although one perhaps fittingly bestowed on molecules of sufficient sophistication to tune thresholds in cell surface receptor signaling ${ }^{3,4}$. The B cell phenotypes reported among mice bearing different $C d 22$ mutations contrast in several immunological parameters. However, there are also notable consistencies indicating CD22 is mainly a negative regulator of $B$ cell immune function. Indeed, multiple autoimmune disease signs have been reported in some CD22-deficient lines. The inhibitory activity of CD22 is attributed to the intracellular ITIM domain and its recruitment of tyrosine phosphatases, such as SHP-1, that downmodulate antigen receptor signaling by reducing phosphotyrosine accumulation.

So why does CD22 have a conserved extracellular Siglec domain? Is it also orchestrating CD22 function? Clearly this domain can induce adhesion among different cells expressing various surface glycoproteins that carry the sialylated glycan ligand of CD22. And yet glycoproteins expressed on B cells, such as CD45 and CD22, are also glycosylated with CD22 ligands. In fact, the Siglec domain of CD22 at the B cell surface is occupied, or 'masked', by endogenous ligands in most contexts. Yet the possibility that Siglec binding function might contribute to immune regulation has been supported by studies of mice rendered deficient in the ST6Gal-I sialyltransferase, a Golgi glycosyltransferase that forms the terminal $\alpha 2,6$ sialic acid linkage essential for CD22 Siglec ligand formation. In the absence of ST6Gal-I, B cell immune dysfunction is evident, with reduced responses after antigen receptor stimulation, including calcium mobilization, phosphotyrosine accumulation and proliferation, as well as a depressed humoral antibody production profile ${ }^{5}$.
'Unmasked' CD22 capable of ligand binding reigns at the B cell surface in ST6Gal-I deficiency. On balance, it is difficult to reconcile the hyperimmune state of CD22 deficiency with the hypoimmune state of ST6Gal-I deficiency in models of CD22 function that incorporate the participation of both Siglec and ITIM domains.

Using a compelling experimental approach to unravel this mystery, Poe et al. have produced selective mutations of CD22 structure in the mouse germline directed toward ablating its Siglec function while maintaining expression on the $\mathrm{B}$ cell surface $^{2}$. Two mutations were engineered separately. One was a truncated CD22 in which the first two N-terminal immunoglobulin-like domains 1 and 2 that encompass the Siglec binding activity (in domain 1) were deleted (CD22 $\Delta 1-2)$. The second CD22 mutant contained two point mutations in which two key arginine residues residing in domain 1 , important for Siglec binding function, were altered to alanine (CD22AA). The immunological outcomes of mice with these mutations were compared with those of mice lacking CD22. The approach was in many ways a success and represents a timely mutagenesis study focused on dissecting the lectin-binding function of a Siglec in an intact mammalian immune system. Poe et al. find that both CD22 $\Delta 1-2$ and CD22AA result in diminution of cell surface CD22 and immunoglobulin $\mathrm{M}$ (IgM), a modest increase in major histocompatibility complex class II expression, a deficiency in marginal zone B cells, an increase in conventional $B$ cells in the peritoneal cavity, increased B cell turnover, enhanced response to stimulation with antibody to CD40 and suboptimal antigen receptor-induced proliferation. In contrast, neither of the two mutations altered B cell migration or tyrosine phosphorylation and SHP-1 recruitment. Discordances emerged in the functional attributes of the two CD22 mutants as well as in comparisons of both mutants to the absence of CD22. The reduction in recirculating bone marrow $\mathrm{B}$ cells in mice lacking CD22 or bearing the CD22AA mutation did not occur in CD22 $\Delta 1-2$ mice. In contrast, the hypermobilization of intracellular calcium that occurs in the absence of CD22 after antigen receptor stimulation failed to take place in $\mathrm{B}$ cells bearing either CD22 $\Delta 1-2$ or CD22AA; instead, the last two mutations conferred normal calcium mobilization profiles.

Can a coherent architecture for CD22 function now be crafted that embraces the Siglec and ITIM domains, one that is consistent

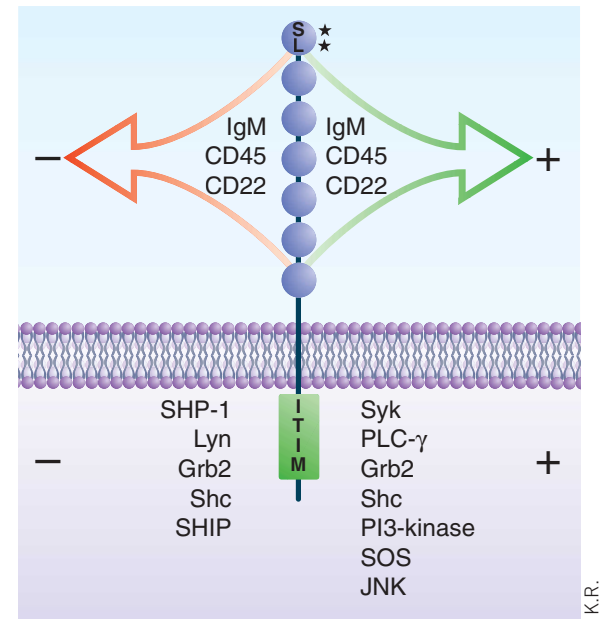

Figure 1 Dynamic and opposing nature of CD22 function. Protein interaction motifs are present in both extracellular and intracellular domains. The extracellular Siglec domain (SL) is the most $\mathrm{N}$-terminal of multiple immunoglobulin-like domains (ovals) and contains conserved arginine residues $\left({ }^{*}\right)$ that participate in glycan binding to sialylated ligands attached to glycoproteins such as the antigen receptor (IgM), CD45 and CD22. The intracellular domain includes ITIM motifs that are tyrosine-phosphorylated after B cell activation and can physically recruit signal transduction molecules as well as influence more distal signaling detected in genetic models. The ability of CD22 to elicit negative (-) and positive $(+)$ signaling in B cell immune function may reflect the identities of molecular partners associated with both of these topologically restricted domains. Simultaneous characterization of both extracellular and intracellular protein interactions in different cell contexts may be required to achieve a mechanistic understanding of CD22 function in molecular detail.

with these and previous findings? The authors admit that the inconsistencies seem to deny this for now. Several contradictions must be resolved, and a closer evaluation of the data leads to further questions. One of the most notable inconsistencies pertains to the involvement of the CD22 Siglec domain in calcium mobilization, which Poe et al. found essentially unaltered by both extracellular mutations. Other groups, using either immortalized B cell lines bearing the same Cd22 mutations or B cells incubated with small molecule CD22 Siglec ligand mimetics, have reported that disruption of Siglec function increases calcium mobilization, similar to $\mathrm{CD} 22$ deficiency ${ }^{6-8}$. As Poe et al. discuss, immortalized B cell lines may yield differences by genetic and contextual influences even when such cells are derived from the same mutant strain of mice. There are also thermodynamic features of CD22 Siglec 
binding that render substantial disruption by sialoside mimetics difficult to achieve. The endogenous CD22 Siglec ligand concentration on the B cell surface has been calculated to be at least $25 \mathrm{mM}$ in the extended region of the glycocalyx and accessible to the extracellular domain of CD22. Even with the low micromolar affinities reported, the concentrations of sialoside ligand mimetics used in published studies are less than $1 \%$ the concentration of endogenous ligand and would achieve the disruption of approximately $10 \%$ of endogenous CD22 Siglec ligand binding 9 . Further characterization of the Siglecdependent effects of these sialioside mimetics would be valuable. And what exactly are the binding properties of the CD22 mutations? The view that they completely abrogate Siglec activity has been derived from very few observations involving cell adhesion and CD45 binding assays ${ }^{10-12}$. It is relevant that CD22 Siglec domain mutations undergo more comprehensive characterization to determine the degree of binding deficit. As Poe et al. point out, discordance between phenotypes of the CD22 $21-2$ and CD22AA mutants may relate to the possibility of asyet-uncharacterized differences in their ligand-binding properties.

Despite these discrepancies, Poe et al. have achieved a milestone in the saga of CD22 function. The next steps are increasingly clear. The authors point out that the involvement of the
CD22 cytoplasmic domain should be evaluated in studies that inactivate its function while retaining cell surface Siglec binding. Another matter pertains to the inconsistencies among CD22 mutant studies that assess a raison d'être of B cell existence: antibody formation. This potential involvement of CD22 needs further evaluation, given reports that include CD22 cell surface redistribution and trans-cellular binding of the Siglec domain with antigenpresenting cells and $\mathrm{T}$ lymphocytes ${ }^{9,13}$. In addition, there are several phenotypic similarities between the CD22 Siglec domain mutants, the CD22 deficiency state and ST6Gal-I depletion. How might the ST6Gal-I-deficient and B cell immune-compromised phenotype relate to CD22 function? The authors suggest the presence of other immunoregulatory lectins that recognize glycan ligands constructed by ST6Gal-I. However, studies that would demonstrate such B cell lectins have yielded evidence for only CD22 (refs. 7,14). Perhaps we do not have a full accounting of CD22 binding mechanisms. Experiments using a modified approach to enhance the detection of colocalized glycoproteins at the cell surface have reported interactions of CD22 with IgM at stoichiometries of $8-16 \%$ that are undiminished by CD22 Siglec domain mutation or after sialic acid depletion. These data suggest that the binding of CD22 to some B cell surface glycoproteins such as the antigen receptor may be mediated by proteinprotein interactions ${ }^{15}$.
Vertebrates have conserved a large genomic investment in glycan variation and recognition processes, and the ability to effectively study the physiological functions of these events is now emerging ${ }^{2,16}$. This should encourage further investments to discern how protein glycosylation and glycan-recognition molecules participate in the formation of immune regulatory signals that emanate from molecular interactions at the cell surface.

1. Crocker, P.R. \& Varki, A. Immunology 103, 137-145 (2001).

2. Poe, J.C. et al. Nat. Immunol. 5, 1078-1087 (2004).

3. Cyster, J. \& Goodnow, C.C. Immunity 6, 509-517 (1997).

4. Tedder, T.F., Tuscano, J., Sato, S. \& Kehrl, J.H. Annu. Rev. Immunol. 15, 481-504 (1997).

5. Hennet, T., Chui, D., Paulson, J.C. \& Marth, J.D. Proc. Natl. Acad. Sci. USA 95, 4504-4509 (1998).

6. Jin, L., McLean, P.A., Neel, B.G. \& Wortis, H.H. J. Exp. Med. 195, 1199-1205 (2002).

7. Kelm, S., Gerlach, J., Brossmer, R., Danzer, C.-P. \& Nitschke, L. J. Exp. Med. 195, 1207-1213 (2002).

8. Danzer, C.-P., Collins, B.E., Blixt, O., Paulson, J.C. \& Nitschke, L. Int. Immunol. 15, 1137-1147 (2003).

9. Collins, B.E. et al. Proc. Natl. Acad. Sci. USA 101 6104-6109 (2004).

10. Engel, P., Wagner, N., Miller, A.S. \& Tedder, T.F. J. Exp. Med. 181, 1581-1586 (1995).

11. Law, C.-L., Aruffo, A., Chandran, K.A., Doty, R.T. \& Clark, E.A. J. Immunol. 155, 3368-3376 (1995).

12. van der Merwe, P.A. et al. J. Biol. Chem. 271, $9273-$ 9280 (1996).

13. Lanoue, A., Batista, F.D., Stewart, M. \& Neuberger, M.S. Eur. J. Immunol. 32, 348-355 (2002).

14. Collins, B.E. et al. Glycobiology 12, 563-571 (2002).

15. Zhang, M. \& Varki, A. Glycobiology advance online publication date, 7 July 2004 (doi:10.1093/glycob/ cwh126).

16. Lowe, J.B. \& Marth, J.D. Ann. Rev. Biochem. 72, 673-691 (2003).

\section{DUBbing down innate immunity}

\section{Neal Silverman \& Katherine Fitzgerald}

\section{The innate inflammatory immune response must be finely tuned to avoid excessive damage to the host. One molecule, A20, is intricately involved in dampening inflammatory signals mediated by both tumor necrosis factor and multiple Toll-like receptors.}

Propral roper control of inflammation is critical to effectively combat pathogens and to maintain homeostasis. In healthy individuals, cytokine production is rapidly activated in response to infection and is downregulated shortly thereafter to prevent the massive systemic response known as shock. A20, by dampening signaling by

Neal Silverman and Katherine Fitzgerald are in the Division of Infectious Disease, Department of Medicine, University of Massachusetts Medical School, Worcester, Massachusetts 01605, USA. e-mail: neal.silverman@umassmed.edu the transcription factor $N F-\kappa B^{1}$, is an important component of this 'shutdown' system. In this issue of Nature Immunology and in a recent issue of Nature, two groups have investigated the molecular mechanism of how A20 downregulates NF- $\kappa B$ activation mediated by tumor necrosis factor receptor (TNFR) and Toll-like receptor (TLR) signaling 2,3 .

The original characterization of A20-deficient mice suggested that A20 specifically inhibited a component of the TNFR1mediated NF- $\kappa \mathrm{B}$ pathway ${ }^{1}$. In Nature Immunology, Ma and colleagues now demonstrate that in addition to its effects on TNF signaling, A20 also downregulates TLR4mediated lipopolysaccharide (LPS) signaling. Mice doubly deficient either in A20 plus TNFR1 or in A20 plus TNF both developed spontaneous inflammation, similar to mice deficient in A20 alone. This shows that A20 regulates TNF-independent proinflammatory signals. In addition, mice reconstituted with A20-deficient hematopoietic cells were hypersensitive to LPS-induced shock, dying within 2 h of LPS injection. Ex vivo, A20deficient macrophages also showed enhanced NF- $\kappa B$ activity in response to TLR2, TLR3 and TLR9 ligands, suggesting that A20 negatively regulates multiple TLR pathways ${ }^{3}$. 